\title{
A review of aortic thrombosis in COVID-19 infection
}

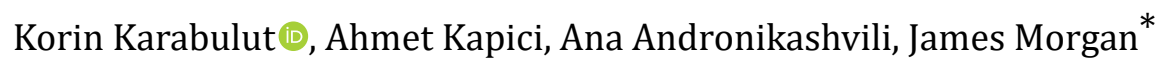 \\ Steward Carney Hospital, Boston, MA 02124, United States \\ *Correspondence: James Morgan, Steward Carney Hospital, 2100 Dorchester Avenue, Boston, MA 02124, United States. \\ James.Morgan@steward.org \\ Academic Editor: Akiko Mammoto, Medical College of Wisconsin, USA
}

Received: March 30, 2021 Accepted: August 8, 2021 Published: October 31, 2021

Cite this article: Karabulut K, Kapici A, Andronikashvili A, Morgan J. A review of aortic thrombosis in COVID-19 infection. Explor Med. 2021;2:410-22. https://doi.org/10.37349/emed.2021.00059

\begin{abstract}
Aim: As the novel coronavirus disease 2019 (COVID-19) pandemic impacts the global healthcare system, evolving data show increased frequency of arterial and venous thromboembolism among patients with COVID-19 infection. Aortic thrombus is a rare thrombotic event with a wide spectrum of clinical manifestations and potential catastrophic complications. This study aimed to elucidate the clinical manifestations, diagnosis and treatment dilemmas of aortic thrombus with COVID-19 infection and raise awareness among frontline medical providers. Aortic thrombosis is rare, but if not considered early in the course of COVID-19 infection, the data suggest that the diagnosis will probably not be made until potentially serious complications arise.

Methods: Literature review was conducted between November 1, 2019, and November 14, 2020, on PubMed and Embase to identify publications regarding aortic thrombosis among COVID-19 cases.

Results: Most of the patients were male with a median age of 67 years, and had comorbidities (most commonly hypertension, dyslipidemia and diabetes mellitus). In our study, underlying atherosclerosis, a common risk factor for aortic thrombus, was identified among $56 \%$ of the patients. Aortic thrombus was symptomatic in $62 \%$ of these patients and most commonly manifested itself as acute limb ischemia (46\%), whereas 30\% of cases were found incidentally during the investigation of elevated inflammatory markers or increased oxygen requirement. Treatment was individualized given the lack of established guidelines for aortic thrombus, including anticoagulation, systemic and catheter directed thrombolysis, and surgical thrombectomy. Overall mortality was found to be $30 \%$ in our study.

Conclusions: Although rare, aortic thrombus has high morbidity and mortality, and can present without any symptoms or underlying aortic disease. Aortic thrombosis is rare, but if not considered early in the course of COVID-19 infection, the data suggest that the diagnosis will probably not be made until potentially serious complications arise.
\end{abstract}

\section{Keywords}

Thrombolysis, thrombectomy, outcomes, surgical, aortic, COVID-19, anticoagulation 


\section{Introduction}

Aortic thrombus is a rare finding as an isolated event. Diagnosis of aortic thrombus is made most often as an incidental finding in the company of other distal thromboembolic sites. Aortic thrombus is typically associated with severe atherosclerosis, embolism, aneurysm, foreign bodies (e.g., graft, stents) and less commonly hypercoagulable, infectious, neoplastic processes, and trauma. Rarely, aortic thrombus has been identified without any underlying risk factor to explain thrombus formation. Given the rarity of disease and absence of randomized controlled trials, there is no consensus on the treatment of aortic thrombi and the management is individualized. Current treatment options include anticoagulation, systemic thrombolysis, endovascular and surgical thrombectomy. In the course of the current pandemic, it has become increasingly clear that coronavirus disease 2019 (COVID-19) increases risk for thrombosis since a marked proportion of patients with severe COVID-19 infection develop venous and arterial thromboembolic complications. Most of the studies report thrombotic complications that are related to deep vein thrombosis and pulmonary embolism; however, data on aortic thrombosis in COVID-19 patients are limited. In our literature review, 43 cases were found to have COVID-19 complicated with aortic thrombus to consolidate evidence of aortic thrombosis in COVID-19 infection and raise awareness. More importantly, we emphasize the importance of making the diagnosis of aortic thrombus, given that, like aortic dissection, if not considered, probably will not be diagnosed early before catastrophic complications arise.

\section{Materials and methods}

Literature review was conducted between November 1, 2019, and November 14, 2020, on PubMed and Embase to identify publications regarding aortic thrombosis among COVID-19 cases [1-26].

\section{Results}

In our literature review of 26 publications, 43 cases with COVID-19 were described to have aortic thrombosis. COVID-19 status was demonstrated with reverse transcriptase polymerase chain reaction and aortic thrombus was diagnosed with computed tomography (CT) angiogram in all cases. A majority of the patients were male: 31 male (72\%), 9 female (20\%), gender of 3 patients was not identified (Figure 1 ). Mean age of the patients was 67 years and only 3 patients were below 50 years of age. The most common comorbidities were hypertension (46.5\%), dyslipidemia (23\%), and diabetes mellitus (20\%). Clinically significant atherosclerotic disease was identified in $20 \%$ of the cases (total of 9 cases: 5 cases with coronary artery disease, 2 cases with stroke, 1 case with transient ischemic attack, 1 abdominal aneurysm with graft). Smoking status as a significant cardiovascular risk factor was reported only in 11 cases (25\%), of which 9 were either smokers or former smokers. A history of thromboembolic disease was reported in only 3 cases; 2 had pulmonary emboli and 1 had deep vein thrombosis. Among the cases in which home medications were available (total of 24), $8.3 \%$ were on anticoagulation for atrial fibrillation; $29.1 \%$ were on antiplatelet medication for various medical indications.

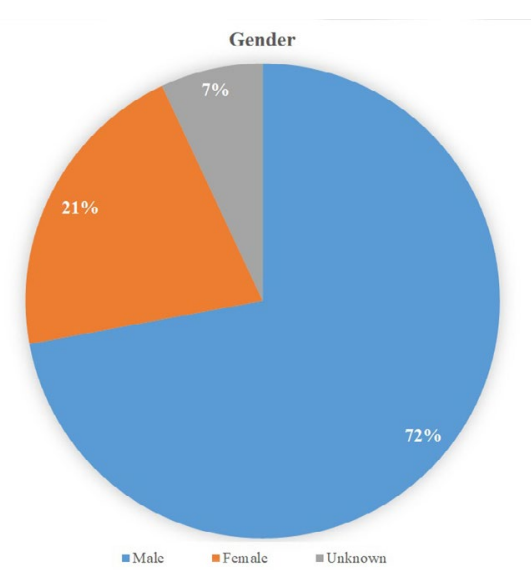

Figure 1. The gender distribution of patients with aortic thrombosis in COVID-19 infection 
At the time of presentation to the hospital, the majority of the cases were admitted for COVID-19 related symptoms (dyspnea, fever, and fatigue). Only 8 cases (18\%) had symptoms of distal arterial thrombosis as an initial chief complaint (ischemic limb in 7 patients and stroke in 1 patient), the remaining cases were discovered later during their hospital course (Figure 2). The primary triggering event for the providers to diagnose the aortic thrombus was symptomatic distally located arterial thrombosis $62 \%, 27$ cases out of 43). In other words, $62 \%$ of cases were found after aortic thrombus embolized distally and caused end-organ damage. However, in the remaining $28 \%$ of the cases, aortic thrombus was found incidentally and fortunately before the thrombus embolized and caused end-organ damage. Among those patients who did not have any symptoms of arterial thromboembolism, CT angiogram was obtained to rule out pulmonary emboli in the presence of high blood levels of inflammatory markers (especially D-dimer) or significant increases in the inflammatory markers over short periods of time, or remarkable changes in oxygen demand.

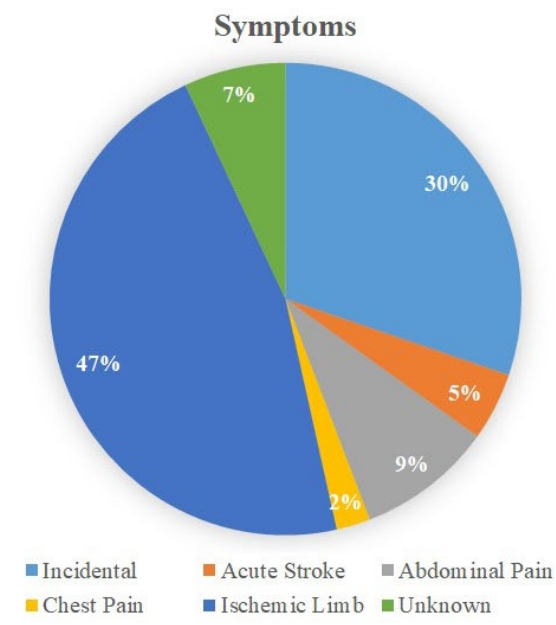

Figure 2. The symptomatology of patients with aortic thrombus in COVID-19 infection

Cases that were complicated by distal arterial events, included limb ischemia (20 patients, 46\% of total cases), acute stroke (4 patients, $9 \%$ of total cases), and visceral artery thrombus resulting in ischemia (4 patients, $9.3 \%$ of total cases). 10 patients (23\%) had multiple emboli affecting more than one territory. In regard to stroke types among the cases, three of them were identified as ischemic stroke but it was unclear if embolic or thrombotic in etiology; the remaining case was an unspecified type of stroke. Even if all of the cases were thromboembolic stroke in etiology, we would not be able to determine definitely the embolus originated from aortic thrombus or other anatomic location. For example, Manghat et al. [22] reported that a patient with recent transcatheter aortic valve replacement (TAVR) and coronary artery bypass grafting (CABG) presenting with chest pain was found to have pulmonary embolism and aortic valve thrombus with embolization to the left anterior descending coronary artery. This patient did not have any COVID-19 related symptoms (dyspnea, cough, fever, etc.), but was found to have severe acute respiratory syndrome coronavirus 2 (SARS-CoV-2) infection on further diagnostics. In $28 \%$ of the cases, aortic thrombus was detected incidentally where CT angiogram was obtained to rule out pulmonary emboli rather than suspecting an arterial event. Associated venous thromboemboli were described in 10 cases (23\%).

Anatomic localization of aortic thrombus varied (Figure 3). The most common thrombus location was abdominal aorta in 13 cases (30\%) followed by descending thoracic aorta in 10 cases (23\%), aortic arch in 7 cases (16\%), aortic bifurcation in 5 cases (11\%) and ascending aorta in 2 cases (4.6\%). Approximately $75 \%$ of the cases had associated arterial thrombus, most commonly seen in the iliac system.

In order to assess the clinical severity, cases were classified to location (i.e. intensive care unit or medical floor), oxygen needs and vasopressor requirements were reported in approximately half of the cases. The majority of the patients were admitted to intensive care units (18 patients, 41\%), whereas 7 patients (16\%) were located on the medical floor. Most of the patients required mechanical ventilation, followed by nasal cannula (1-4 L/min), and a few patients were placed on either high flow, nonrebreather oxygen masks or 
non-invasive ventilation. Vasopressor requirement was observed in $50 \%$ of the patients, however this was not re-ported in most of the cases. Of the available data, 25 patients (58\%) had favorable outcomes whereas 11 patients (30\%) expired. Information was limited regarding concomitant atherosclerosis, only 34.8\% $(n=15)$ of the total patients had available information. From available data, 8 out of 15 patients, 53.3\% had underlying atherosclerosis. Among those patients, one patient had accompanying aortic aneurysm and another one had concomitant aortic stenosis.



Figure 3. The anatomic distribution of aortic thrombus among patients COVID-19 infection

Laboratory tests were significant for elevation of the inflammatory markers. Fibrinogen levels were $>4 \mathrm{~g} / \mathrm{L}$ in 15 patients, D-dimer levels were $>3 \mathrm{mg} / \mathrm{L}$ in 23 patients. Interleukin (IL)-6 levels were $>5 \mathrm{ng} / \mathrm{L}$ in 10 cases. Assays for antithrombin III, proteins $\mathrm{C}$ and $\mathrm{S}$, fibrinogen, and antiphospholipid antibodies had been performed only in 6 cases (14\%) and antiphospholipid antibodies were found in 2 cases. However, hypercoagulability laboratory tests can be falsely positive in acute illness especially with acute thromboembolic events. Therefore, hypercoagulability tests should be repeated after resolution of the thromboembolic event in or-der to find evidence of an underlying hemostatic disorder.

Treatment approaches varied among the patients (Figures 4-6), 60.4\% $(n=26)$ of the patients were treated conservatively, $37.2 \%(n=16)$ of the patients treated with variable invasive approaches, whereas $2.3 \%(n=1)$ of patient's treatment was not reported. Among patients treated with conservative modalities; anticoagulation alone was preferred in $32.5 \%(n=14)$, anticoagulation with antiplatelet therapy was used in $6.9 \%(n=3)$, and systemic thrombolysis was performed in $4.6 \%(n=2)$. The treatment modality was reported as medical therapy (unknown details) among $16.2 \%(n=7)$. Invasive treatment modalities were applied to $37.2 \%$ of the patients: surgical thrombectomy was performed in $20.9 \%(n=9)$, axillobifemoral bypass surgery was chosen in $4.6 \%(n=2)$, catheter directed thrombolysis in $4.6 \%(n=2)$, catheter directed thrombolysis with surgical thrombectomy in 2.3\% $(n=1)$, endovascular thrombectomy $2.3 \%(n=1)$ and systemic thrombolysis with thrombectomy in $2.3 \%(n=1)$. Of the available data for the total cases (Table 1), 25 patients (58\%) had favorable outcomes where-as 11 patients (30\%) died. Comparing the treatment modalities, among patients treated conservatively, outcome was favorable in $57.6 \%(n=15)$ and the mortality rate was $19.2 \%(n=5)$. By contrast, in patients undergoing invasive treatment approaches, favorable outcome was reported for $62.5 \%$ $(n=10)$; the mortality rate was $25 \%(n=4)$. Only $16 \%(n=7)$ of the patients had repeat CT angiogram for the evaluation of residual thrombus; of those treated with anticoagulation only, $42.7 \%(n=3)$ had complete resolution. Partial improvement was ob-served in $57.1 \%(n=4)$ of the patients, whom were treated with various invasive modalities respectively; thrombectomy $(25 \%, n=1)$, systemic thrombolysis $(25 \%, n=1)$, catheter directed thrombolysis $(25 \%, n=1)$, catheter directed thrombolysis with thrombectomy $(25 \%, n=1)$. Death was observed within a very short period of time after the aortic thrombus was found in $36.3 \%$ of the cases. However, we are unable to definitely conclude aortic thrombus as a major reason of mortality even 
for those cases, as most of them had multiple comorbidities with extensive medical illnesses. CT angiogram images of aortic thrombus in COVID-19 cases are shown in Figures 7 and 8.

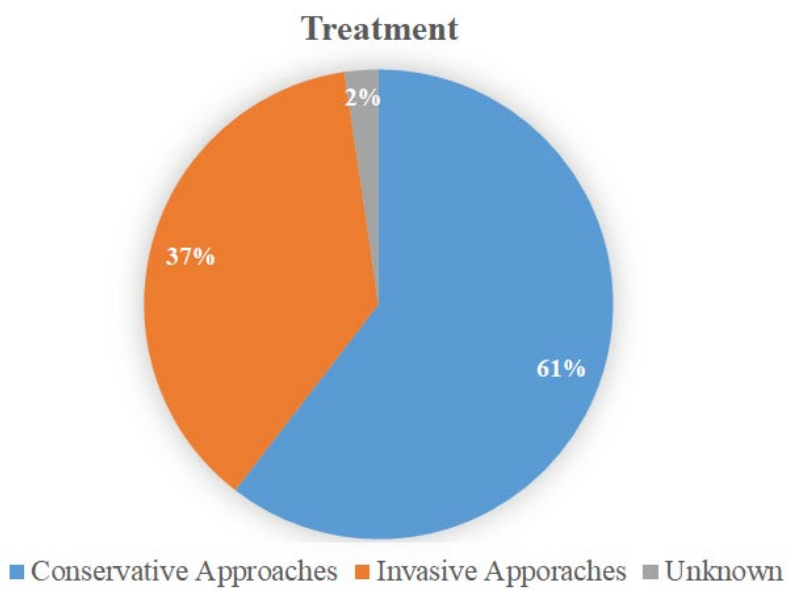

Figure 4. The treatment modalities used for aortic thrombus with COVID-19 infection

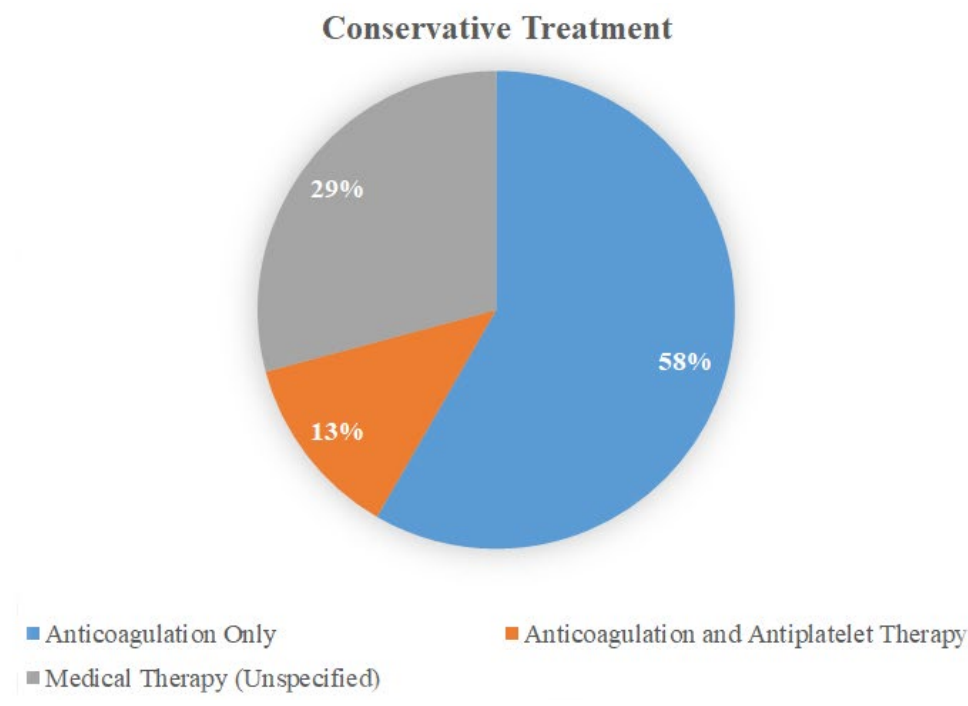

Figure 5. The non-invasive treatment modalities used for aortic thrombus with COVID-19 infection

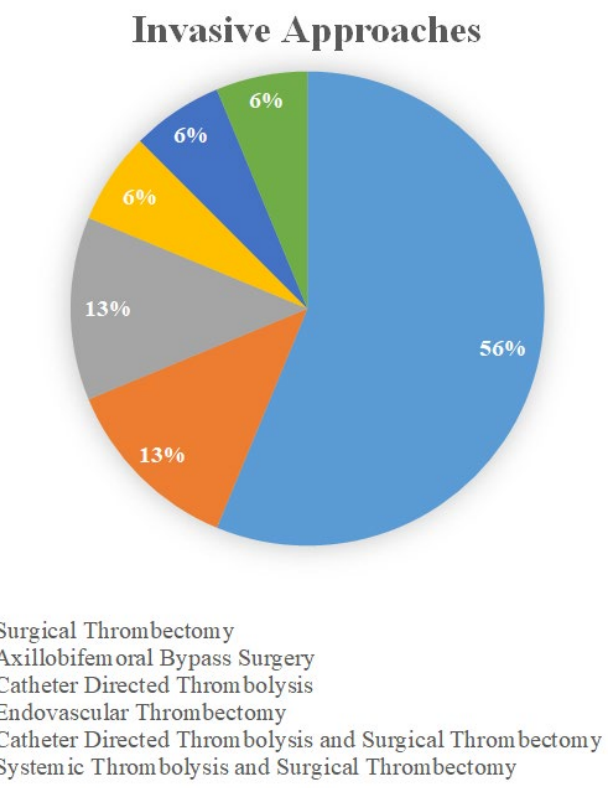

Figure 6. The invasive treatment modalities used for aortic thrombus with COVID-19 infection 
Table 1. Summary of various treatment modalities utilized for patients with COVID-19 infection complicated with aortic thrombus and overall outcomes

\begin{tabular}{llll}
\hline Treatment modality & Favorable outcome & Unknown outcome & Mortality \\
\hline Anticoagulation only $(n=14,32.5 \%)$ & $57 \%(n=8)$ & $7 \%(n=1)$ & $35 \%(n=5)$ \\
Anticoagulation with antiplatelet therapy $(n=3,6.9 \%)$ & $100 \%(n=3)$ & $0 \%$ & $0 \%$ \\
Medical management (not reported specifically) $(n=7,16.2 \%)$ & $42 \%(n=3)$ & $28 \%(n=2)$ & $28 \%(n=2)$ \\
Systemic thrombolysis $(n=2,4.6 \%)$ & $50 \%(n=1)$ & $50 \%(n=1)$ & $n=0$ \\
Surgical thrombectomy $(n=9,20.9 \%)$ & $77.7 \%(n=7)$ & $n=0$ & $22.3 \%(n=2)$ \\
Axillobifemoral bypass surgery $(n=2)$ & $50 \%(n=1)$ & $n=0$ & $50 \%(n=1)$ \\
Catheter directed thrombolysis $(n=2,4.6 \%)$ & $50 \%(n=1)$ & $50 \%(n=1)$ & $n=0$ \\
Endovascular thrombectomy $(n=1,2.3 \%)$ & $n=0$ & $100 \%(n=1)$ & $n=0$ \\
Catheter directed thrombolysis with surgical thrombectomy $(n=1$, & $100 \%(n=1)$ & $n=0$ & $n=0$ \\
2.3\%) & & $n=0$ & $100 \%(n=1)$ \\
Systemic thrombolysis with surgical thrombectomy $(n=1,2.3 \%)$ & $n=0$ & & \\
\hline
\end{tabular}

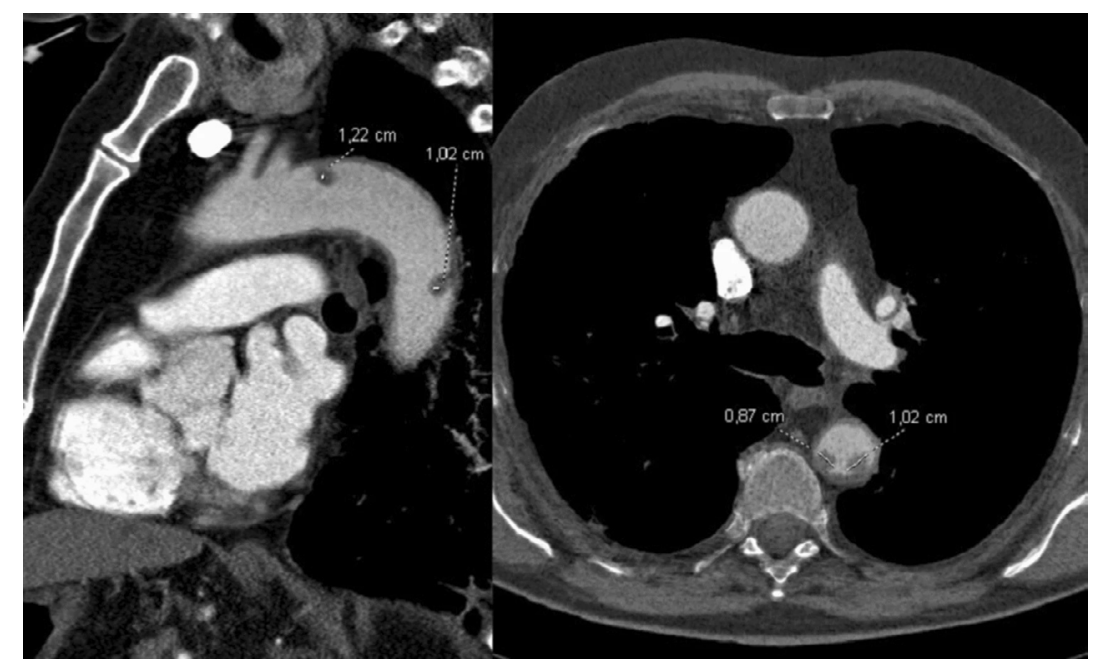

Figure 7. Floating aortic thrombi in a patient with COVID-19 infection

Note. Reprinted with permission from "Aortic thrombus in patients with severe COVID-19: review of three cases," by de Carranza M, Salazar DE, Troya J, Alcázar R, Peña C, Aragón E, et al. J Thromb Thrombolysis. 2021;51:s237-42 (https://doi.org/10.1007/ s11239-020-02219-z). (OSpringer
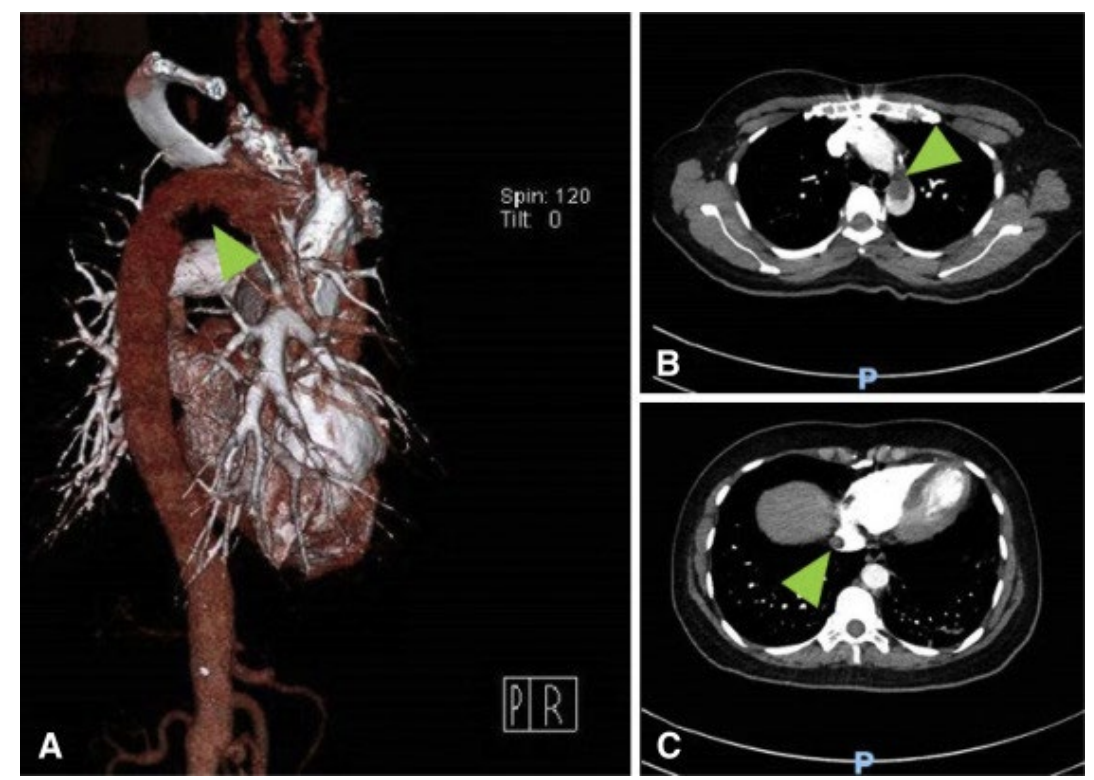

Figure 8. (A) CT with three dimensional reconstruction of the aortic arch with a filling defect (arrow); (B) coronal view of the chest, with a large thrombus present in the aortic arch (arrow); (C) CT of the chest with a thrombus seen in the suprahepatic portion of the inferior vena cava (arrow)

Note. Reprinted with permission from "Aortic Arch Thrombus and Pulmonary Embolism in a COVID-19 Patient," by Gandotra P, SupariwalaA, Selim S, Garra G, Gruberg L. JEmerg Med. 2021;60:223-5 (https://doi.org/10.1016/j.jemermed.2020.08.009). (OElsevier 


\section{Discussion}

Aortic thrombus is a rare diagnosis and is typically associated with severe atherosclerosis, aneurysm, foreign bodies, and less commonly hypercoagulable, infectious, neoplastic processes, and trauma [27-29]. In this study, we focused aortic thrombosis in patients with COVID-19, aiming to raise suspicion for a very likely association.

Multiple studies suggest that there is an increased risk of thromboembolic events in COVID-19 patients. According to the study by Klok et al. [30], the incidence of thromboembolic complications ranges from 5\% to $15 \%$ in critically ill patients without COVID-19, whereas incidence of thromboembolic complications in critically ill patients with COVID-19 is $35-45 \%$, which supports the hypothesis of increased risk of thromboembolic complications in COVID-19.

Emerging evidence suggests that COVID-19 increases the risk of thromboembolism by multiple mechanisms. According to the current data, COVID-19 results in a hypercoagulable state characterized by micro- and macro-vascular thrombotic angiopathy [31, 32]. Microvascular thrombotic depositions were seen in small vessels of the lungs and other organs on post-mortem examination of patients with COVID-19 infection [33]. Pro-inflammatory cytokines are increased with severe COVID-19 infection, such as tumor necrosis factor- $\alpha$ (TNF- $\alpha$ ) and IL (particularly IL-1 and IL-6), which results in activation of the coagulation cascade as well as inhibition of endogenous anticoagulant pathways [34]. IL-6 as a key factor in cytokine storm, also promotes megakaryopoiesis which provokes thrombosis in the microcirculation [35]. Diffuse endothelial inflammation and evidence of direct viral infection of the endothelial cells were demonstrated in pathology specimens of patients with COVID-19 infection [36]. Type 3 hypersensitivity reaction of endothelial walls consisting of antigen-antibody complex-es with fibrinoid necrosis were observed with COVID-19 infection [37]. High antiphospholipid antibody levels are postulated as a pathway for hypercoagulation by enhancing platelet adhesion to fibrin and endothelium, and have been observed with COVID-19 infection [38-40]. ACE2 is the responsible receptor for the viral adhesion on endothelial cells and viral replication results in inflammatory cell infiltration, endothelial cell apoptosis, and microvascular prothrombotic effects [36]. This may explain the cerebrovascular events and myocardial ischemia seen in younger patients with COVID-19. In addition, hyperviscosity is reported in COVID-19. Fibrinogen is one of the major determinants of blood viscosity, and high levels are seen in patients with COVID-19. Hyperviscosity directly predisposes to thrombosis and induces endothelial dysfunction [41]. On the other hand, SARS-CoV-2 does not appear to be associated with significant bleeding unlike some of other RNA viruses such as Ebola and hemorrhagic fever viruses. According to the article by Connors et al. [42] "the SARS-CoV-2 virus does not appear to have intrinsic procoagulant effects itself, although more information is needed."

Data regarding pre-existing atherosclerosis was limited in our study, 8 out of 15 cases (53\%) had concomitant atherosclerosis. Given that atherosclerosis is a major risk factor for aortic thrombus, COVID-19 likely played a role with its increased thrombotic complications for $47 \%$ of the cases. A meta-analysis by Fayad et al. [43] which was published in 2013, included 200 patients with aortic mural thrombus in a normal or minimally atherosclerotic aorta. Similar to our study, the most common comorbidity was hypertension (22\%). Furthermore, the study found that the most common mode of presentation was limb ischemia (84\%), followed by visceral ischemia (27\%) and stroke (14\%) which was similar to our study findings. Importantly in this study, there was no incidentally found aortic thrombus case, which differs from our study (30\% of cases were incidentally found in our study). Another meta-analysis [44] which reviewed 90 patients with COVID-19 who had arterial thrombosis also showed fewer incidentally found cases with thromboembolism (3\%) compared to our study. This raises a significant suspicion that COVID-19 might be causing more silent aortic thrombosis compared to non-COVID patients. Therefore, due to its complications resulting in severe morbidity and mortality, healthcare professionals should be more vigilant to search for aortic thrombus with a lower threshold for imaging, particularly in the setting of COVID-19 infection.

One of the most typical findings in patients with COVID-19 is an elevated D-dimer level [42], which is associated with increased mortality. In an observational study [45] in 183 patients with COVID-19, a mean D-dimer concentration in non-survivors $(2.12 \mathrm{mg} / \mathrm{L})$ was higher than in survivors $(0.61 \mathrm{mg} / \mathrm{L})$. Furthermore, 
another study [46] showed that D-dimer levels greater than $1 \mathrm{mg} / \mathrm{L}$ on admission were associated with 18-times increased risk of death (95\% Confidence Interval 2.6-128.6; $P=0.0033)$. In our study, all patients with available data on D-dimer (38 patients, $82 \%$ ) had levels greater than $1 \mathrm{mg} / \mathrm{L}$, which supports the approach to have a lower threshold for imaging. Unfortunately, there is no definitive laboratory cut-off value that necessitates imaging studies. Diagnosis of aortic thrombus remains challenging and is mainly based on clinical impression due to its rarity as well as the lack of significant guidelines for diagnostic approach. Therefore, in light of the findings in our study and review of the COVID-19 literature, we highly recommend obtaining imaging studies (i.e. CT angiogram) particularly on the patients with elevated D-dimer even with the slightest clinical suspicion, and even in the absence of signs or symptoms of distal arterial thromboembolism, in order to make an early diagnosis and initiate treatment before serious complications occur. This recommendation is supported by a considerable number of incidental aortic thrombus cases in COVID-19 patients, the significantly increased thromboembolism risk due to COVID-19, and the markedly higher mortality in patients with elevated D-dimer. In the presence of contraindications to CT angiography (e.g., renal failure, contrast allergy), alternative options include magnetic resonance angiography (MRA), transesophageal echocardiography (TEE), intravascular ultrasound (IVUS), duplex ultrasound and traditional angiography. Prophylactic hydration with normal saline might be considered if there are concerns for renal failure; anti-histamines may be considered if there are concerns for allergic reactions. Empiric anticoagulation might be considered as an option for high risk patients in the setting of hemodynamic instability. On the other hand, obtaining CT pulmonary angiogram (CTPA) for every patient with elevated D-dimer level can be unfeasible given the high-risk in hemodynamically unstable patients, particularly those with underlying kidney injury. Recent studies suggest that the safety of CTPA for screening venous thromboembolic events would be more increased by, applying age-adjusted or pre-test probability adjusted D-dimer thresholds or increasing the threshold for D-dimer to $1000 \mathrm{mg} / \mathrm{dL}$ [47-52]. Abrupt worsening of respiratory status, hemodynamic instability (hypotension and/or tachycardia), unilateral limb swelling, signs of right-heart strain on electrocardiogram or bedside echocardiogram, increasing D-dimer levels over time, intravenous line malfunction or increase in dead space can alarm clinicians to suspect venous thromboembolic events (VTE) in COVID-19 patients, similar triggers for obtaining CTPA was found in our review as well [47, 53, 54]. However, most of those studies investigated the value and feasibility of screening (VTE) and did not include arterial events and importantly they were limited by small sample size and lack of prospective validation. Thus, more studies are needed to be done to establish a dedicated screening protocols for both arterial and venous thrombosis in COVID-19 patients. Because of significant number of asymptomatic aortic thrombosis have been detected in the literature, we suggest a CTPA be obtained if the D-dimer is elevated or an unexpected clinical change in the COVID-19 patients.

Current data on aortic thrombosis associated with COVID-19 are limited to case reports and case series. Therefore, in order to obtain more accurate data on incidence and mortality of aortic thrombosis in COVID-19, the articles including arterial thrombosis with COVID-19 were also reviewed. A recent systematic review [44] revealed the incidence of arterial thrombus ranged from 2.7-8\% in severe/critically ill COVID-19 patients. Most of the cases were symptomatic (97\%), ischemic limb being the most common presentation. Unfortunately, the definite number of cases with aortic thrombus was not reported separately. Nineteen percent of the arterial thrombosis cases included involvement of the great vessels (aorta, common iliac, common carotid, and brachiocephalic trunk). The mortality rate of the arterial thromboembolism was found to be approximately $20 \%$ in this study. It is important to note that this study mainly focused on the arterial thromboembolism in COVID-19 in general, rather than aortic thrombosis. Therefore, the higher number of symptomatic events in this study compared to our review likely results from the difference in the main focus of the studies and the fact that aortic thrombosis itself could have more indolent clinical manifestations compared to distal arterial thrombosis. According to another study [55] which was conducted prior to the COVID-19 pandemic, autopsy reports showed $0.45 \%$ incidence of aortic thrombosis in the general population, with $17 \%$ showing evidence of distal embolization and $6 \%$ of sufficient severity to be considered the cause of death. Thus, despite the rarity of aortic thrombosis, it is associated with high mortality and morbidity which should raise high suspicion for prompt diagnostic consideration to prevent catastrophic complications. 
Given the rarity of disease, absence of randomized controlled trials and treatment guidelines, treatment of aortic thromboembolism must be individualized. Current treatment options include anticoagulation, thrombolysis (systemic or catheter directed), endovascular and surgical thrombectomy.

Unfortunately, review of the current literature did not include any significant data on the treatment and follow up of the patients with COVID-19 who had aortic thrombus. Thus, we reviewed studies regarding aortic thrombus in general. A meta-analysis conducted in 2013 [43], compared two treatment approaches: anticoagulation (112 patients) and aortic surgery (88 patients). Persistence or recurrence of the thrombus was significantly more common in the anticoagulation group. Patients in the anticoagulation group tended to have more ischemic complications, however the difference was not statistically significant and the mortality rates were similar between the two groups. This study favored a surgical approach over anticoagulation treatment alone. Another study [56] in 2017 focused on descending thoracic aortic thrombus compared three different treatment modalities: anticoagulation (26 patients), open surgery (19 patient), and thoracic endovascular aortic repair (TEVAR) (29 patients). Medical anticoagulation therapy was associated with higher rates of persistent thrombus compared with open thrombectomy or TEVAR. Importantly, there was no thrombus found recurrent in the TEVAR group, suggesting TEVAR as an effective therapy in patients with suitable thrombus anatomy without prohibitive clot burden. On the other hand, many other studies recommend anticoagulation as the preferred primary treatment for aortic thrombus [57-60]. Complete resolution of thrombus with anticoagulation was reported in multiple studies [57, 61]. Unfortunately, only a few case reports $[62,63]$ included thrombolytic therapy for the treatment of aortic thrombus, which was not included in the meta-analyses and no comparison is available to the other treatment options. The treatment of choice for aortic thrombosis, is still on a case-by-case basis for non-COVID-19 patients. Considering the novelty of COVID-19 and rarity of aortic thrombosis, treatment approach for aortic thrombosis with COVID-19 needs further studies. Choosing between surgical options and minimal invasive options with interventional radiology, special considerations would include degree of ischemia, threat of the end organ damage, anatomic location, accessibility and clot burden.

In light of the studies we reviewed, we recommend prompt initiation of systemic anticoagulation in all patients after the diagnosis of aortic thrombus, absent critical contraindication to these agents, such as active blood loss. In addition, since the data from recent studies favor surgical and endovascular approaches due to fewer thrombus recurrence rates is promising, open aortic surgery may be a more appropriate initial therapy in patients with a large clot burden. Particularly in younger patients with fewer comorbidities, a surgical approach would be feasible. Additionally, TEVAR, being a minimally invasive and low risk procedure with lower embolism recurrence, can be used successfully for patients with aortic thrombus presenting with initial or recurrent embolization, and patients who have a contraindication or treatment failure to anticoagulant therapy.

In conclusion, COVID-19 infection is associated with increased risk of thromboembolic complications including arterial events, such as aortic thrombosis. Aortic thrombus is a very rare clinical entity and there are no clear treatment guidelines in the literature. Although the majority of the patients with aortic thrombus present with clear ischemic symptoms, a significant number were found to have incidental thrombus. Therefore, high clinical suspicion is needed for evaluation of thromboembolic events in COVID-19 patients, which can prompt earlier treatment. Anticoagulation is recommended in all patients with aortic thrombosis in the absence of contraindications. Additional treatment options include surgical and endovascular approaches as well as thrombolysis. Although it is very difficult to conduct prospective randomized studies given the rarity of the disease, further studies are needed to establish a clear treatment guideline for aortic thrombus. Additionally, most of the data were gathered from the studies prior to the current COVID-19 pandemic. Since the dynamics of thromboembolism in COVID-19 might be different from the normal population, additional data, including treatment and follow up regarding aortic thrombosis in COVID-19 patients, are needed. 


\section{Abbreviations}

COVID-19: Coronavirus Disease 2019

CT: computed tomography

CTPA: computed tomography pulmonary angiogram

TEVAR: thoracic endovascular aortic repair

\section{Declarations}

\section{Author contributions}

JM and KK contributed conception and design of the study; KA and AA organized the database; AA performed the statistical analysis; KK wrote the first draft of the manuscript; AA wrote results section, JM, KK and AA wrote introduction and discussion sections. JM provided overall supervision for this study. All authors contributed to manuscript review, and read and approved the submitted version.

\section{Conflicts of interest}

The authors declare that they have no conflicts of interest.

\section{Ethical approval}

Not applicable.

\section{Consent to participate}

Not applicable.

\section{Consent to publication}

Informed consent to publication was obtained from relevant participants.

\section{Availability of data and materials}

Data can be provided upon request from the responding author.

\section{Funding}

Not applicable.

\section{Copyright}

(c) The Author(s) 2021.

\section{References}

1. Le Berre A, Marteau V, Emmerich J, Zins M. Concomitant acute aortic thrombosis and pulmonary embolism complicating COVID-19 pneumonia. Diagn Interv Imaging. 2020;101:321-2.

2. Kashi M, Jacquin A, Dakhil B, Zaimi R, Mahé E, Tella E, et al. Severe arterial thrombosis associated with Covid-19 infection. Thromb Res. 2020;192:75-7.

3. de Carranza M, Salazar DE, Troya J, Alcázar R, Peña C, Aragón E, et al. Aortic thrombus in patients with severe COVID-19: review of three cases. J Thromb Thrombolysis. 2021;51:237-42.

4. Vulliamy P, Jacob S, Davenport RA. Acute aorto-iliac and mesenteric arterial thromboses as presenting features of COVID-19. Br J Haematol. 2020;189:1053-4.

5. Mullan C, Powierza C, Miller PE, Geirsson A, Vallabhajosyula P, Assi R. Spontaneous coronavirus disease 2019 (COVID-19)-associated luminal aortic thrombus. J Thorac Cardiovasc Surg. 2020;160:e13-4.

6. Azouz E, Yang S, Monnier-Cholley L, Arrivé L. Systemic arterial thrombosis and acute mesenteric ischemia in a patient with COVID-19. Intensive Care Med.2020;46:1464-5. 
7. Vacirca A, Faggioli G, Pini R, Teutonico P, Pilato A, Gargiulo M. Unheralded lower limb threatening ischemia in a COVID-19 patient. Int J Infect Dis. 2020;96:590-2.

8. Silingardi R, Gennai S, Migliari M, Covic T, Leone N. Acute limb ischemia in COVID-19 patients: could aortic floating thrombus be the source of embolic complications? J Vasc Surg. 2020;72:1152-3.

9. Mosbahi S, Heinisch PP, Schoenhoff FS, Berger DC. COVID-19-associated aortic arch thrombus. Eur J Cardiothorac Surg. 2020;58:870.

10. Mahan K, Kabrhel C, Goldsmith AJ. Abdominal pain in a patient with COVID-19 infection: a case of multiple thromboemboli. Am J Emerg Med. 2020;38:2245.e3-5.

11. Giacomelli E, Dorigo W, Fargion A, Calugi G, Cianchi G, Pratesi C. Acute thrombosis of an aortic prosthetic graft in a patient with severe COVID-19-related pneumonia. Ann Vasc Surg. 2020;66:8-10.

12. Gomez-Arbelaez D, Ibarra-Sanchez G, Garcia-Gutierrez A, Comanges-Yeboles A, Ansuategui-Vicente M, Gonzalez-Fajardo JA. COVID-19-related aortic thrombosis: a report of four cases. Ann Vasc Surg. 2020;67:10-3.

13. Baeza C, González A, Torres P, Pizzamiglio M, Arribas A, Aparicio C. Acute aortic thrombosis in COVID-19. J Vasc Surg Cases Innov Tech. 2020;6:483-6.

14. Coscas R, Coggia M. Acute aortic thrombosis revealing a COVID-19 infection. Eur J Vasc Endovasc Surg. 2020;60:827.

15. Katchanov J, Kalisch J, Herzing W, Knorr F, Havla M, Klink T, et al. Extensive aortic thrombosis in a patient with COVID-19. Ann Emerg Med. 2020;76:373-4.

16. Woehl B, Lawson B, Jambert L, Tousch J, Ghassani A, Hamade A. 4 cases of aortic thrombosis in patients with COVID-19. JACC Case Rep. 2020;2:1397-401.

17. Gandotra P, Supariwala A, Selim S, Garra G, Gruberg L. Aortic arch thrombus and pulmonary embolism in a COVID-19 patient. J Emerg Med. 2021:60:223-5.

18. Masana M, Martinez LI, Gil M, Bueno G, Llagostera S. Thoracic aortic mural thrombus, right ventricular clot and pulmonary embolism in a patient with COVID-19 pneumonia. Vasc Endovascular Surg. 2021;55:273-6.

19. Naudin I, Long A, Michel C, Devigne B, Millon A, Della-Schiava N. Acute aortoiliac occlusion in a patient with novel coronavirus disease-2019. J Vasc Surg. 2021;73:18-21.

20. Lari E, Lari A, AlQinai S, Abdulrasoul M, AlSafran S, Ameer A, et al. Severe ischemic complications in Covid-19—a case series. Int J Surg Case Rep. 2020;75:131-5.

21. Mukherjee A, Ghosh R, Furment MM. Case report: COVID-19 associated renal infarction and ascending aortic thrombosis. Am J Trop Med Hyg. 2020;103:1989-92.

22. Manghat NE, Hamilton MCK, Joshi NV, Vohra HA. Acute postoperative thrombosis of an aortic valve prosthesis and embolic myocardial infarction in a coronavirus disease 2019 (COVID-19)-positive patient—an unrecognized complication. JTCVS Tech. 2020;4:111-3.

23. Wengerter SP, Wengerter KR, Masoudpoor H, Sagarwala A, Karim O, Rao N, et al. Acute aortoiliac and infrainguinal arterial thrombotic events in four patients diagnosed with the novel coronavirus 2019. J Vasc Surg Cases Innov Tech. 2020;6:698-702.

24. Patel P, Yu Y, Zia S, Padberg F, Curi M, Huang J. Systemic thrombolysis as initial treatment of COVID-19 associated acute aortoiliac and lower extremity arterial thrombosis. Ann Vasc Surg. 2021;70:297-301.

25. Muhammad K, Tantawy TG, Makar RR, Olojugba O. Successful catheter-directed thrombolysis for acute lower limb ischemia secondary to COVID-19 infection. Ann Vasc Surg. 2021;71:103-11.

26. Kenizou D, Perrin C, Harzallah I, Bresson D, Allimant P, Calcaianu M, et al. Multiple arterial thrombosis in a 78-year-old patient: catastrophic thrombotic syndrome in COVID-19. CJC Open. 2021;3:198-200.

27. Mando R, Gemayel R, Chaddha A, Barbat JJ, Cami E. Peripherally embolizing aortic thrombus: the workup, management, and outcome of primary aortic thrombus. Case Rep Cardio. 2019;2019:8132578. 
28. Laperche T, Laurian C, Roudaut R, Steg PG. Mobile thromboses of the aortic arch with-out aortic debris. A transesophageal echocardiographic finding associated with unexplained arterial embolism. Circulation. 1997;96:288-94.

29. Morris ME, Galiñanes EL, Nichols WK, Ross CB, Chauvupun J. Thoracic mural thrombi: a case series and literature review. Ann Vasc Surg. 2011;25:1140.e17-21.

30. Klok FA, Kruip MJHA, van der Meer NJM, Arbous MS, Gommers DAMPJ, Kant KM, et al. Incidence of thrombotic complications in critically ill ICU patients with COVID-19. Thromb Res. 2020;191:145-7.

31. Henry BM, Vikse J, Benoit S, Favaloro EJ, Lippi G. Hyperinflammation and derangement of renin-angiotensin-aldosterone system in COVID-19: a novel hypothesis for clinically suspected hypercoagulopathy and microvascular immunothrombosis. Clin Chim Acta. 2020;507:167-73.

32. Spiezia L, Boscolo A, Poletto F, Cerruti L, Tiberio I, Campello E, et al. COVID-19-related severe hypercoagulability in patients admitted to intensive care unit for acute respiratory failure. Thromb Haemost. 2020;120:998-1000.

33. Levi M, Thachil J, Iba T, Levy JH. Coagulation abnormalities and thrombosis in patients with COVID-19. Lancet Haematol. 2020;7:e438-40.

34. Huang C, Wang Y, Li X, Ren L, Zhao J, Hu Y, et al. Clinical features of patients infected with 2019 novel coronavirus in Wuhan, China. Lancet. 2020;395:497-506.

35. Roncati L, Ligabue G, Nasillo V, Lusenti B, Gennari W, Fabbiani L, et al. A proof of evidence supporting abnormal immunothrombosis in severe COVID-19: naked megakaryocyte nuclei increase in the bone marrow and lungs of critically ill patients. Platelets. 2020;31:1085-9.

36. Varga Z, Flammer AJ, Steiger P, Haberecker M, Andermatt R, Zinkernagel AS, et al. Endothelial cell infection and endotheliitis in COVID-19. Lancet. 2020;395:1417-8.

37. Roncati L, Ligabue G, Fabbiani L, Malagoli C, Gallo G, Lusenti B, et al. Type 3 hyper-sensitivity in COVID-19 vasculitis. Clin Immunol. 2020;217:108487.

38. Roncati L, Manenti A, Manco G, Farinetti A, Mattioli A. The COVID-19 arterial thromboembolic complications: from inflammation to immunothrombosis through antiphospholipid autoantibodies. Ann Vasc Surg. 2021;72:216-7.

39. Bowles L, Platton S, Yartey N, Dave M, Lee K, Hart DP, et al. Lupus anticoagulant and abnormal coagulation tests in patients with Covid-19. N Engl J Med. 2020;383:288-90.

40. Cavalli E, Bramanti A, Ciurleo R, Tchorbanov AI, Giordano A, Fagone P, et al. Entangling CoVID-19 associated thrombosis into a secondary antiphospholipid antibody syndrome: diagnostic and therapeutic perspectives (Review). Int J Mol Med. 2020;46:903-12.

41. Ahmed S, Zimba O, Gasparyan AY. Thrombosis in coronavirus disease 2019 (COVID-19) through the prism of Virchow's triad. Clin Rheumatol. 2020;39:2529-43.

42. Connors JM, Levy JH. COVID-19 and itfs implications for thrombosis and anticoagula-tion. Blood. 2020;135:2033-40.

43. Fayad ZY, Semaan E, Fahoum B, Briggs M, Tortolani A, D'Ayala M. Aortic mural thrombus in the normal or minimally atherosclerotic aorta. Ann Vasc Surg. 2013;27:282-90.

44. Cheruiyot I, Kipkorir V, Ngure B, Misiani M, Munguti J, Ogeng'o J. Arterial thrombosis in coronavirus disease 2019 patients: a rapid systematic review. Ann Vasc Surg. 2021;70:273-81.

45. Tang N, Bai H, Chen X, Gong J, Li D, Sun Z. Anticoagulant treatment is associated with decreased mortality in severe coronavirus disease 2019 patients with coagulopathy. J Thromb Haemost. 2020;18:1094-9.

46. Zhou F, Yu T, Du R, Fan G, Liu Y, Liu Z, et al. Clinical course and risk factors for mortality of adult inpatients with COVID-19 in Wuhan, China: a retrospective cohort study. Lancet. 2020;395:1054-62. 
47. Koleilat I, Galen B, Choinski K, Hatch AN, Jones DB, Billett H, et al. Clinical characteristics of acute lower extremity deep venous thrombosis diagnosed by duplex in patients hospitalized for coronavirus disease 2019. J Vasc Surg Venous Lymphat Disord. 2021;9:36-46.

48. van der Hulle T, Cheung WY, Kooij S, Beenen LFM, van Bemmel T, van Es J, et al; YEARS study group. Simplified diagnostic management of suspected pulmonary embolism (the YEARS study): a prospective, multicentre, cohort study. Lancet. 2017;390:289-97.

49. Kearon C, de Wit K, Parpia S, Schulman S, Afilalo M, Hirsch A, et al; PEGeD Study Investigators. Diagnosis of pulmonary embolism with d-dimer adjusted to clinical probability. N Engl J Med. 2019;381:2125-34.

50. Righini M, Van Es J, Den Exter PL, Roy PM, Verschuren F, Ghuysen A, et al. Age-adjusted D-dimer cutoff levels to rule out pulmonary embolism: the ADJUST-PE study. JAMA. 2014;311:1117-24.

51. Choi JJ, Wehmeyer GT, Li HA, Alshak MN, Nahid M, Rajan M, et al. D-dimer cut-off points and risk of venous thromboembolism in adult hospitalized patients with COVID-19. Thromb Res. 2020;196:318-21.

52. Stals M, Kaptein F, Kroft L, Klok FA, Huisman MV. Challenges in the diagnostic approach of suspected pulmonary embolism in COVID-19 patients. Postgrad Med. 2021;133:36-41.

53. Rosovsk RP, Sanfilippo KM, Wang TF, Rajan SK, Shah S, Martin KA, et al. Anticoagulation practice patterns in COVID-19: a global survey. Res Pract Thromb Haemost. 2020;4:969-83.

54. Garcia-Olivé I, Sintes H, Radua J, Abad Capa J, Rosell A. D-dimer in patients infected with COVID-19 and suspected pulmonary embolism. Respir Med. 2020;169:106023.

55. Machleder HI, Takiff H, Lois JF, Holburt E. Aortic mural thrombus: an occult source of arterial thromboembolism. J Vasc Surg. 1986;4:473-8.

56. Meyermann K, Trani J, Caputo FJ, Lombardi JV. Descending thoracic aortic mural thrombus presentation and treatment strategies. J Vasc Surg. 2017;66:931-6.

57. Bowdish ME, Weaver FA, Liebman HA, Rowe VL, Hood DB. Anticoagulation is an effective treatment for aortic mural thrombi. J Vasc Surg. 2002;36:713-9.

58. Choukroun EM, Labrousse LM, Madonna FP, Deville C. Mobile thrombus of the thoracic aorta: diagnosis and treatment in 9 cases. Ann Vasc Surg. 2002;16:714-22.

59. Hahn TL, Dalsing MC, Sawchuk AP, Cikrit DF, Lalka SG. Primary aortic mural thrombus: presentation and treatment. Ann Vasc Surg. 1999;13:52-9.

60. Tsilimparis N, Hanack U, Pisimisis G, Yousefi S, Wintzer C, Rückert RI. Thrombus in the non-aneurysmal, non-atherosclerotic descending thoracic aorta- an unusual source of arterial embolism. Eur J Vasc Endovasc Surg. 2011;41:450-7.

61. Berneder S, van Ingen G, Eigel P. Arch thrombus formation in an apparently normal aorta as a source for recurrent peripheral embolization. Thorac Cardiovasc Surg. 2006;54:548-9.

62. Krüger T, Liske B, Ziemer S, Lindemann S, Ziemer G. Thrombolysis to treat thrombi of the aortic arch. Clin Appl Thromb Hemost. 2011;17:340-5.

63. Durdil V, Fiedler J, Alan D, Vejvoda J, Veselka J. Multiple mobile aortic thrombosis treated by thrombolysis. A case report. J Thromb Thrombolysis. 2007;24:315-6. 